\title{
Death by Stimulation of the Left Vagus Nerve in a Case of Neck Grasping in a Healthy Young Man
}

\author{
Saverio Potenza ${ }^{1}$, Alessandro Mauro Tavone ${ }^{2}$, Claudia Scipione ${ }^{3}$, Giovanni Arcudi ${ }^{4}$, Gian Luca Marella \\ ${ }^{1}$ Researcher of Legal Medicine, ${ }^{2}$ Resident Forensic Pathologist, ${ }^{3}$ Resident Forensic Pathologist, University of \\ Rome "Tor Vergata", Department of Biomedicine and Prevention, via Montpellier 1, 00166 Rome, Italy, ${ }^{2}$ Andrea \\ Attanasio, Indipendent Researcher, ${ }^{4}$ Dean of School of Medicine and Surgery, University Our Lady Of Good \\ Counsel, Rr. Dritan Hoxha, Tiranë, Albania, 5Professor of Legal Medicine, University of Rome "Tor Vergata", \\ Department of Surgical Sciences, via Montpellier 1, 00166 Rome, Italy
}

\begin{abstract}
Fatal neck pressure due to manual strangulation is a relatively common event. Sudden deaths that occur in the first seconds of pressure are attributed to effects of the nerve. According to leading authors, the process occurs through a reflex arc where the afferent (sensory) nerve impulses emerge in the carotid complex of the nerve endings, but not in the vagus trunk itself.

On the contrary, we describe one such fatality. The body of a man in his early twenties came to attention in our Institute. According to testimonies and investigations, the victim had a domestic fight with his brother. After a verbal altercation, the victim was grabbed by the neck and immediately fell dead. During the external examination, only two small finger-nail superficial abrasions were found in the left cervical region. No other signs of violence were detected. The dissection of the neck only revealed a perineural hematoma in the left vagus trunk in the cervical tract. The cause of death was attributed to neurally mediated cardiac arrest induced by direct left vagus nerve compression.
\end{abstract}

Keywords: Vagus nerve direct stimulation; Sudden death; Fatal pressure of the neck; Forensic diagnosis.

\section{Introduction}

Fatal pressure on the neck provides one of the most complex and controversial areas of "asphyxial" deaths, as the mechanism is often uncertain. To understand the mechanisms resulting in death caused by pressure on the neck, several anatomical and physiological factors must be considered such as airway occlusion, occlusion of the neck veins, compression of the carotid arteries and nerve effects. ${ }^{1}$

The last few mentioned are considered to be the ones with the most controversial medical-legal aspects.

In fact, the absence of certain diagnostic method for deaths carried out exclusively by the effects on the nerve represent a diagnosis excluding any clear signs that may indicate other causes of death. There is further controversy in the medical field as to which real pathways and reflex arcs are capable of this type of rapid death.
The physiological role of nerve transmission carried out by the vagus nerve is well known. While at the clinical level, its direct stimulation is recognized to produce powerful effects both on humans and guinea pigs that are useful in therapies including bradycardic, arrhythmic and even cardiac arrest. ${ }^{2,3}$ Many authors believe that in cases of fatality for short and even slight neck pressure, "nervous effects" are fundamentally determinant by a reflex arc in which the afferent sensory part is conveyed by the carotid complex, but not by the vagal trunk itself, excluding the possibility of an activation in response to direct mechanical stimuli in its cervical tract. ${ }^{1}$

We report, however, a case of fatal pressure of the neck in which we believe that the mechanism which determined death was precisely that of direct stimulation of the vagal trunk.

Case History: A young man in his early twenties died at home. According to witnesses and police reports, 
the victim was involved in a domestic dispute with his brother who, after a verbal dispute, grabbed him around the neck with his right hand.

Immediately following this, the victim collapsed to the ground lifeless.

No immediate attempts for resuscitation were made, and upon arriving at the scene, the victim was pronounced dead.

Autopsy Findings: During the external inspection of the corpse, two small finger-nail abrasions were found in the skin of the left cervical region (fig. 1).

Upon dissecting the neck planes, a hemorrhagic infiltration was detected surrounding and infiltrating the left vagus nerve in its tract below the common carotid bifurcation, in correspondence to the skin abrasions (fig. 2).

Autopsies for the thoracic, abdominal, skull and brain structures all appeared normal.

The histological examination did not reveal anything significant, and the toxicology report was negative.

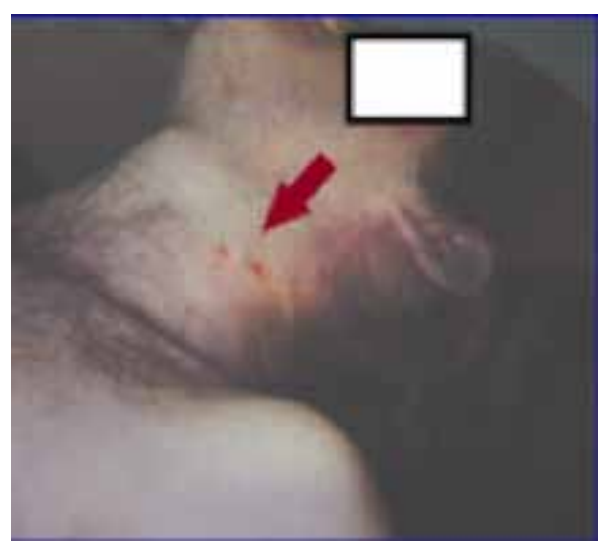

Figure 1. Two finger-nail abrasions

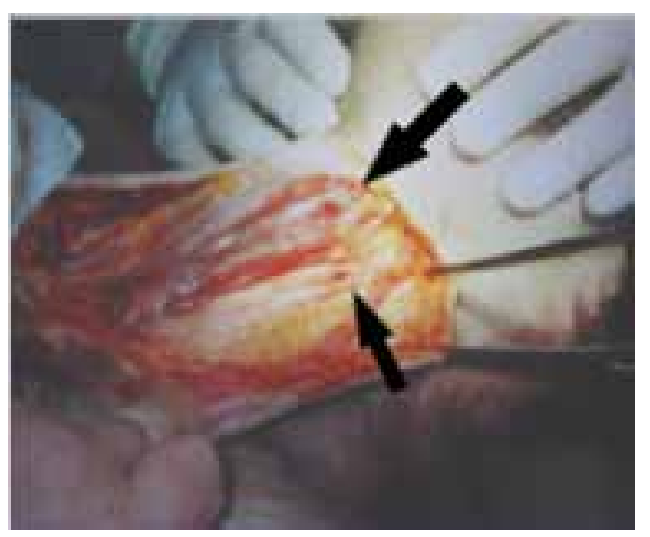

Figure 2. Perineural hemorrhagic infiltration

\section{Discussion}

While it is reported that a mild or 'playful' pressure applied to the neck may have induced an 'instantaneous death' related to a hypokinetic arrhythmia and cardiac arrest due to 'vagal inhibition', which is a well-known mechanism involving stimulation of the carotid sinuses, ${ }^{4}$ the same cannot be said about the direct stimulation of the vagus nerve itself.

Sudden death due to direct vagus nerve stimulation during fatal neck compression is a very rare occurrence, and only few cases have been reported in literature. $5,6,7$

We believe that the physiopathological theory behind those deaths is the direct mechanical activation of a parasympathetic efferent pathway of the carotid sinus reflex arc,via the vagus nerve and the parasympathetic ganglia, to the heart, but without the activation of the stretch receptor of the carotid sinus and the stimulation of the vagal nuclei in the medulla.

For these reasons, as the carotid sinus massage (CSM) or pressure, following the same efferent limb, may lead to a cardio-inibithory response, defined as 3 seconds or longer of ventricular standstill or asystole, ${ }^{8}$ the same would happen if the vagus is directly and mechanically activated.

Although the possibility of this kind of activation is uncertain, two considerations must be taken:

1. a condition of carotid sinus hypersensitivity $(\mathrm{CSH})$ is well known clinically and leads to the cardioinhibitory response to the CSM. It is known to be asymptomatically quite common in the population and should not be omitted in such an abnormal event. $^{9}$

2. cases of severe bradycardia or asystole due to direct stimulation of the vagus nerve or its branches have been directly observed during neck surgery and reported. ${ }^{10,11}$

In order to solve this case and reach our conclusions, we carefully performed a post-mortem examination, finding only two small finger-nail abrasions on the neck of the victim in the absence of any additional external signs of violence. In addition, during the internal exam, the only significant sign observed was the perineural hematoma of the left vagus trunk, indicating there was a significant compression of the nerve. 
This illustrates the importance of an accurate postmortem examination in forensic pathology. To avoid the risk of misdiagnosing the cause of death, all of the wounds, even the ones that seem most irrelevant, must be evaluated accordingly to their severity and location.

Based on the results and evidence from the postmortem examination, we believe that the victim's sudden death was indeed due to an Instantaneous Neurogenic Cardiac Arrest (INCA) induced by direct vagus nerve compression.

Fatal vagal inhibition following a direct vagus nerve injury is considered a very rare occurrence, especially those with left vagus compression considering the anatomical and physiopathological reasons.

The efferent cholinergic fibers represent the parasympathetic innervations of the heart that slows the heart rate. The right vagus branch innervates the sinoatrial node, the normal pacemaker of the heart. The left vagus only innervates atrial muscle fibers and its stimulation has generally limited effects which are predominantly directed at the atrial muscle only slowing down the electric conduction to the AV node. ${ }^{12}$

We believe that the left inhibitory parasympathetic activation related death is derived from cholinergic overstimulation resulting in a severe atrioventricular block and cardiac arrest.

\section{Conclusion}

Deaths for direct manual vagus stimulation are such rare events. The mechanisms involved are uncertain as uncertain is the real possibility of that happening.

We described a case in which we retain this event as the explanation. We propose a physiopatological theroy involving the activation of the only efferent pathway of the carotid sinus reflex and that must consider a condition of increased sensibility to direct stimulation.

Also, for the relative fewer effect on the heart of left vagusin comparison to the contralateral, our case seems such a rarer occurrence, linked to a severe AV block due to decreased electrical conduction in atrial muscle cells.

The importance of a careful necroscopic examination, well evaluating also the apparently irrelevant factors, must be clear in similar cases.
Declarations of Interest: The Authors declare that there is no conflict of interest.

Ethical Clearance: The following study didn't need an ethical clearance from any committee.

\section{References}

1. Saukko P., Knight B. Knight's Forensic Pathology. 4th ed. Chapter 15. CRC Press; 2015

2. Harumi Hotta, Jason Lazar, Rena Orman, Kiyomi Koizumi, Kanako Shiba, Haroon Kamran, Mark Stewart,Vagus nerve stimulationinduced bradyarrhythmias in rats. Autonomic Neuroscience,Volume 151, Issue 2,2009, Pages 98-105,

3. Garamendi-Ruiz I, Gómez-Esteban JC. Cardiovascular autonomic effects of vagus nerve stimulation. Clin Auton Res. 2019 Apr; 29(2):183194.

4. Robert M. Berne, Matthew N. Levy: Principles of Physiology, $2^{\text {nd }}$ edition. Mosby, 1996

5. Watanabe M, Unuma K, Fujii $Y$, Noritake K, Uemura K. An autopsy case of vagus nerve stimulation following acupuncture. Leg Med (Tokyo). 2015 Mar;17(2):120-2.

6. Barranco R, Molinelli A, Ventura F. An Unusual Murder: Vasovagal Death From Atypical Stabbing to the Neck. Am J Forensic Med Pathol. 2018 Dec; 39(4):367-369.

7. Patel F. Vasovagal death from screwdriver stabbing of the neck. J Clin Forensic Med. Volume 5, Issue 4,1998, Pages 205-206,ISSN 1353-1131,

8. Moya A, Sutton R, Ammirati F, Blanc JJ, Brignole M, Dahm JB, Deharo JC, Gajek J, Gjesdal K, Krahn A, Massin M, Pepi M, Pezawas T, Ruiz Granell R, Sarasin F, Ungar A, van Dijk JG, Walma EP, Wieling W. Guidelines for the diagnosis and management of syncope (version 2009). Eur Heart J. 2009 Nov;30(21):2631-71.

9. Thomas JE. Hyperactive carotid sinus reflex and carotid sinus syncope. Mayo Clin Proc. 1969 Feb; 44(2):127-39.

10. Almquist M, Thier M, Salem F. Cardiac arrest with vagal stimulation during intraoperative nerve monitoring. Head Neck. 2016 Apr; 38(S1): E2419-E2420. 
11. A. Frenke: A Nearly Lethal Screw: An Unusual Cause of Recurrent Bradycardia and Asystole Episodes after Fixation of the Cervical Spine. Case Reports in Critical Care. Volume Oct 2017, Article ID 3748930 .
12. Howland RH. Vagus Nerve Stimulation. Curr Behav Neurosci Rep. 2014 Jun; 1(2):64-73. 